\title{
Relations between ER Stress, UPR and Cancer Biology
}

\author{
Yixuan Wang ${ }^{\mathrm{a}, 1 \mathrm{st}}$, Jinrui Huang ${ }^{\mathrm{b}, 1 \mathrm{st}}$, Siqi Xiong ${ }^{\mathrm{c}, 1 \mathrm{st}}$ \\ ${ }^{a}$ Luoyang No. 1 high school, Luoyang Henan, China, 471000.kristinwang426@yahoo.com \\ ${ }^{b}$ Indiana Area Senior High, Indiana PA, United States, 15701.jrhuang1225@gmail.com \\ ${ }^{c} 18$ Meadow Pl, Tustin CA, United States,92782. siqix3@uci.edu
}

\begin{abstract}
Effects of external factors such as protein homeostasis can trigger the production of endoplasmic reticulum stress, which can be restored by the unfolded protein response (UPR). The UPR plays a central role in the development of cancer biology, and it is also associated with various malignant characteristics, such as invasion and metastasis. Malignant cells can thrive under various conditions, such as hypoxia, which limits their ability to produce protein-folding, and this impairs their ability to generate an anti-tumor immune response. Studies have also revealed that the effects of ER stress on the development of cancer immunity are significant, this concept proposes that the use of targeted drugs to address this issue could help improve the efficacy of currently available cancer immunotherapies. In this paper, we discussed the impact of ER stress and UPR on cancer treatment, and their implications with cancer biology. We concluded that UPR induced by ER stress in cancer cells has the ability to mutate and use this feature to play its own advantages. This is another reason why UPR can be regarded as an anti-cancer treatment mechanism.
\end{abstract}

Keywords: Cancer, ER Stress, Immunotherapy, UPR, Microenvironment

\section{INTRODUCTION}

Previous studies have predicted that prolonged ER Stress contributes to the development of many diseases. The most common among which is the malignant diseases, including breast cancer, lung cancer, colon cancer, and leukemia. Endoplasmic Reticulum is the cell organelle that provides a unique environment for protein folding, assembling, and bond formation prior to exposure to extracellular environments. Synthesis rate of secretory cells is extremely high in the ER [1]. To achieve such an overwhelming task without losing quality of the produced proteins, the cell spends large amounts of energy ensuring and maintaining homeostasis. When this balance is disrupted, the capacity of ER to continue folding proteins becomes overwhelmed, unfolded proteins are detained and thus leading to the unfolded protein response. UPR, also known as the Unfolded Protein Response, is a series of signal transduction cascades triggered by disruption of homeostasis in the ER lumen, such as when the number of unfolded proteins exceeds the capacity of the ER function. The UPR is ultimately a regulatory mechanism that enhances the expression of proteins that are involved in the function of ER. These proteins include: chaperone proteins and ERrelated degradation components. Chaperones are a group of proteins that help proteins fold. ER-related degradation components can eliminate misfolded proteins. Due to this ability, UPR induced by ER stress leads to the promotion of tumor cell proliferation, metastasis, chemoresistance, and angiogenesis. Which are all major problems in the biology study field. Further investigation into ER stress' impact on cell function would provide more development as to how cancer cells survive and proliferate.

\section{ANALYSIS OF RELATIONS BETWEEN ER STRESS, UPR AND CANCER BIOLOGY}

In past tumor observational studies, UPR signal plays an important role in tumor research and treatment. In a common trend in previous research, UPR markers are increased to indicate ER stress and protein translation shifts in hypoxic tumors. However, the latest results show that UPR can cause cancer directly, [2] without the traditional concept of misfolded protein. Solid Tumors often show elevated levels of ER partners, including GRP78 and GPR94. Increased Probable G-protein coupled receptor 78 expression is closely associated with increased proliferation rate and invasiveness. Emerging concepts suggest that UPR has much unexpected relevance and role in cancer research. This paper provides an overview of the UPR signaling pathway, with 
particular emphasis on new research findings in relation to malignant diseases.

UPR is an adaptive mechanism produced by cells in the process of fighting against cancer. It triggers cell transformation and metabolic state through regulating mechanisms, so as to improve cell survival rate. UPR signaling regulates the interaction between oncogenes and suppressor genes. The interdependence and interference between these signaling pathways are vital to the human body, mis regulation of these pathways can result in serious consequences. UPR activation at different stages of cancer progression is far more complex than previously found. The new discovery that prolonged UPR activation due to unresolved ER stress leads to cell death further complicates the search for cancer therapy. UPR prevents oncogene-induced malignant progression. In both cases, transformed cells identified as those that survived the crisis of oncogeneinduced apoptosis showed high levels of UPR activation when ER stress was not triggered by external factors. Currently there are three known proximal sensors of the UPR, these include the PERK, ATF6, and IRE1 [1]. The sensors all associate with BiP in their inactive form, and once unfolded protein accumulates, the sensors are released and activated simultaneously. ATF6 is cleaved by proteases $\mathrm{S} 1 \mathrm{P} / \mathrm{S} 2 \mathrm{P}$ in the Golgi, the fragment of ATF6 migrates to the nucleus. IRE1 and PERK are both phosphorylated, then the phosphorylated IRE1 induces splicing of XPB1 mRNA, which would produce a more potent transcription factor [1,3]. And PERK would phosphorylate eIF2 which proceeds to slow the general translation rate. The combination of these three sub pathways induces expression of genes that produce proteins to increase the ER folding capacity. At the same time, ERAD would be accelerated to remove misfolded proteins in the lumen. Most studies suggest that UPR pathways are regulated in a highly specific manner to meet complex developmental and metabolic demands.

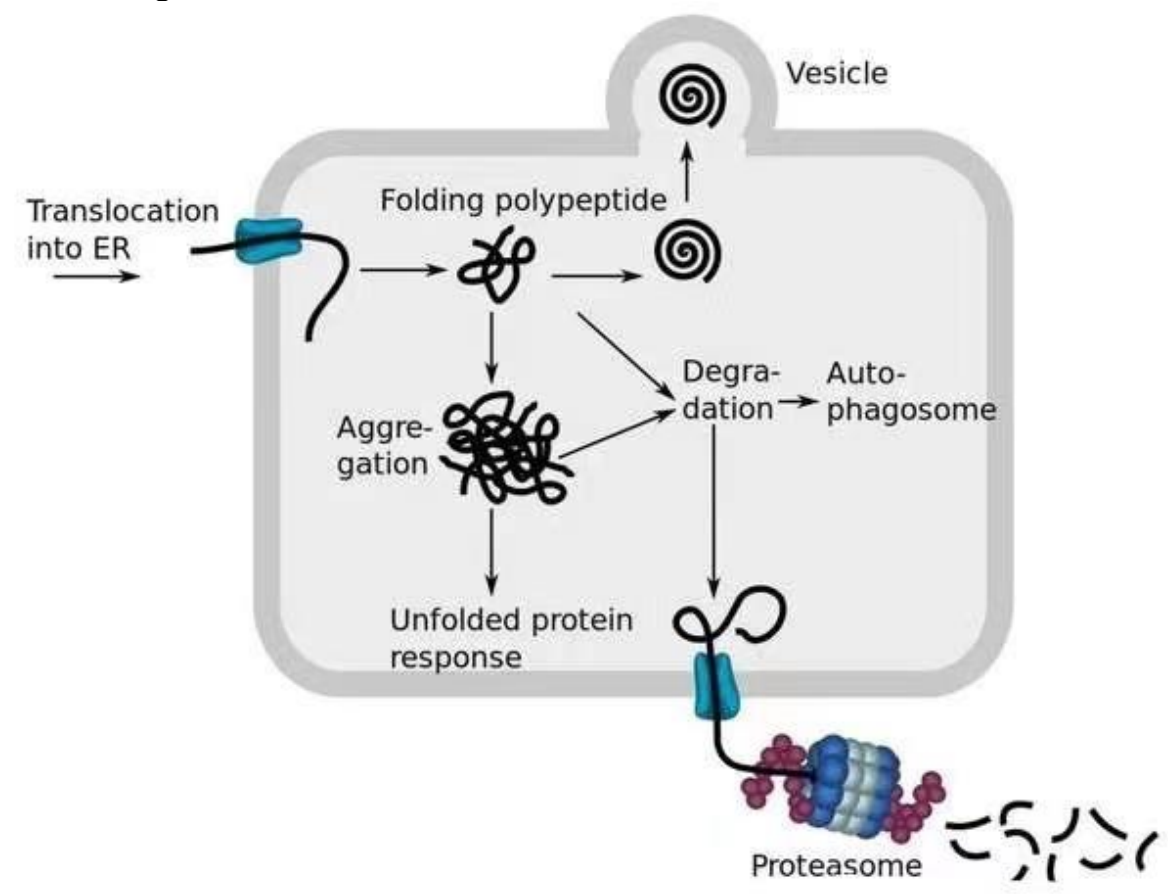

Figure 1. (Simplified Process of UPR)

The ER strictly regulates processes that handles, modifies, and folds proteins. These processes are crucial to the cell's survival and function, any abnormality that causes a malfunction in the ER can result in dramatic consequences. In tumor type cells, a variety of transcriptional, oncogenic, and metabolic mutations lead to the emergence of hostile environments that corrupts the functioning of ER. Multiple factors in the tumor microenvironment (TME) interactively disrupts the capability of the Endoplasmic Reticulum to properly fold proteins in the malignant cells. Several main factors include: hypoxia, nutrient deprivation, reactive oxygen species, and low levels of $\mathrm{pH}$, which we will discuss in a more detailed fashion in the next section.
Hypoxia refers to the condition of having low levels of oxygen in blood streams and tissues. This may result from high altitudes and heart conditions that affect the proper pumping of blood throughout the body. Hypoxia is a common feature in TME which disrupts homeostasis in ER and induce stress in this compartment [2]. Oxygen is an important component in the post translational processes, lack of oxygen restricts the functions of oxygen-dependent compartments in the ER which is required for disulfide bond formation and protein folding [2]. Oxygen is also mandatory in the process of lipid desaturation. Lack of oxygen would lead to decreased content of desaturated lipids which would reduce ER expansion and therefore decrease protein folding capacity. 
It should be noted that only extreme hypoxia can result in such conditions, modest hypoxia (1-5\%) have rarely any effects on UPR activation. This phenomenon raises the question of whether ER stress and UPR can be regulated in an orderly way.

Nutrient deprivation is another environmental factor that can affect ER stress in cells. The amount of nutrients available is often related to how well the metabolic processes are being performed. Insufficient excessive nutrient supply can cause metabolic stress which disrupts ER homeostasis and lead to ER stress. The lack of vital energy sources such as glucose and glutamine can damage many biosynthetic pathways which most often are involved in protein folding processes. One major biosynthetic pathway is the production of ATP, which operates as a phosphate donor required for protein folding in ER [2]. Reactive oxygen species are highly reactive chemicals formed from oxygen. Protein folding in the ER is heavily dependent on these redox reactions. Accumulation of ROS intracellularly in the ER in response to external conditions can disturb homeostasis in ER. Excessive accumulation of the amount of ROS intracellularly can alter calcium channels in ER can skew the capacity of the compartment to fold proteins [2, 4]. One of the major energy pathways used by cancerous cells is aerobic glycolysis. Glycolysis produces Latic which would lower the surrounding $\mathrm{pH}$. Proton sensing receptors can be activated upon detection of low $\mathrm{pH}$ and therefore trigger several responses of the UPR, likely by disrupting intracellular calcium homeostasis and inducing ROS overproduction [2].

The above factors can provoke a state of consistent ER stress that has been shown to demonstrate protumoral features in cancerous cells while reprogramming the function of adaptive immune cells. Based on this, activation of ER stress sensors has been identified as key regulators of tumor growth and metastasis as well as chemotherapy and immunotherapy.

\section{CONCLUSION}

From the review of the current literature, a variety of diseases are associated with the long-term occurrence of ER stress, especially malignant diseases. In this case, it can be concluded that UPR activation induced by ER stress is related to the progression of malignant diseases.[5] Research shows that components of the UPR can cause the promotion of cancer and aggravation of the condition. In cancer biology, UPR promotes cancer development in various ways, and data suggests that UPR can improve tumor cell survival along with drug resistance, helping them adapt to changing environments [6]. In addition, the need for protein production caused by oncogene triggers an overall ER stress that activates the three sensor branches of UPR. Our structural studies show the precise process of how ER stress can trigger the UPR. During prolonged ER stress, UPR response as chaperones to assist protein folding. The three known sensors, PERK, ATF6 and IRE1, are associated with BiP prior to activation. Once released, the sensors are activated respectively as the unfolded proteins accumulate in the endoplasmic reticulum lumen [1]. Fragment of ATF6 migrates to nucleus after being cleaved. IRE1 and PERK are phosphorylated, and the former causes the XBP1 bind to mRNA, and PERK perform elf2 phosphorylation resulting in slow translations. With these three pathways, UPR induces protein production and increase ER folding capacity [4]. UPR plays an important role in tumor transformation and proliferation. Oncogenic transformation is a multi-step process that uses UPR to surmount various obstacles. Over-activation of MYC in normal epithelial cells produces a big amount of proteotoxic stress resulting in decreased cell viability. Nevertheless, in a variety of cancers, including lymphoma, neuroblastoma, prostate cancer and breast cancer, cells subjected to MYC induced stress show increase UPR activity. Thus, fully activated UPR is essential to stress adaptation caused by MYC driven oncogene transformation. 


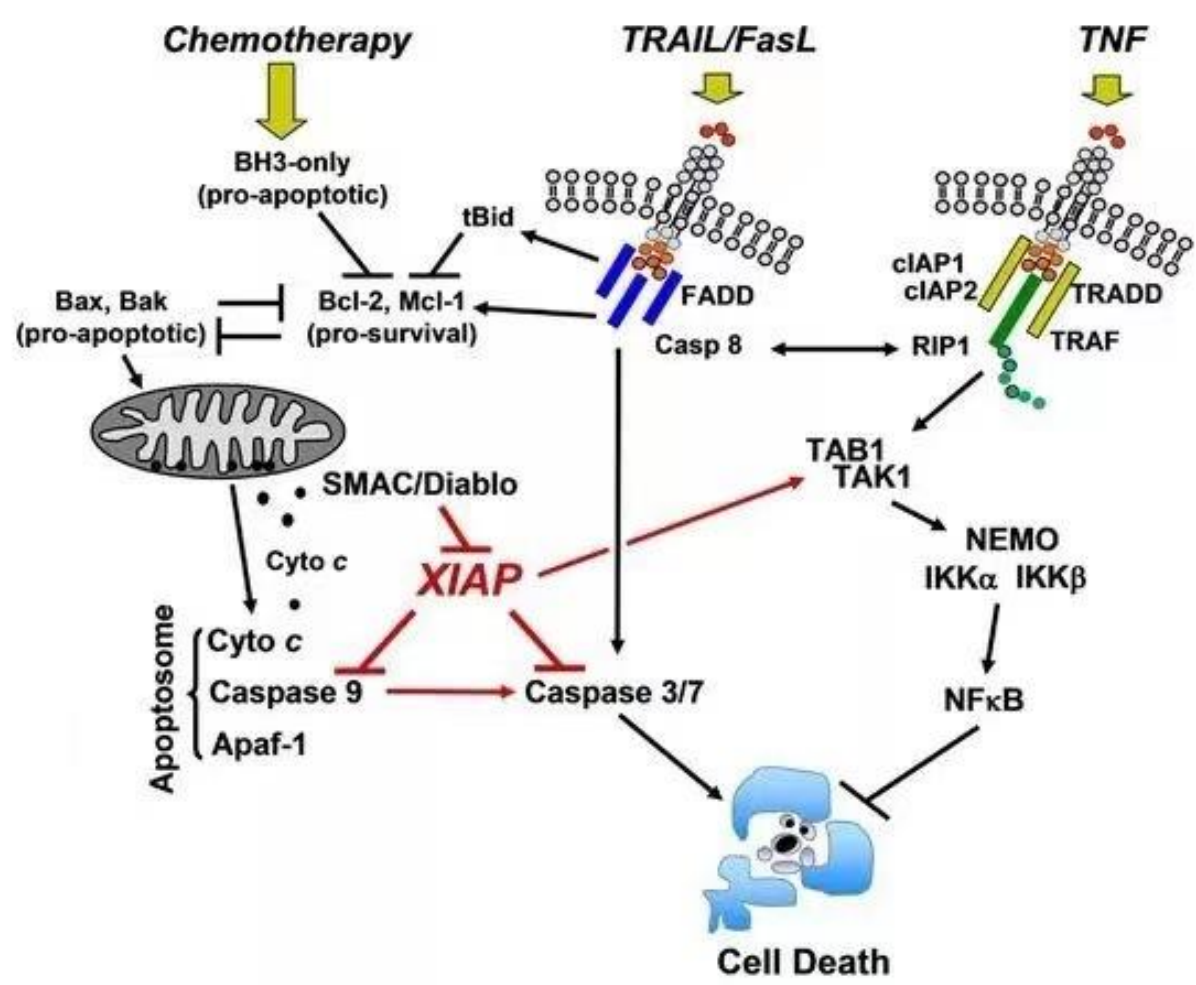

Figure 2. (Apoptotic Pathway)

Another crucial idea in cancer biology: the concept of targeted therapy is a mile stone in this area. Most targeted therapies treat cancer by interfering with specific proteins that promote tumor growth and spread throughout the body. Targeted therapy can treat cancer in many different ways. They include helping the immune system fight cancer cells, preventing cancer cell proliferation, blocking signals related to angiogenesis, delivering cytotoxic substances to cancer cells, and so on. This paper aims for the studies in cancer biology area where UPR are involved with the promotion of tumor cells, and we should target future research on how to keep normal cells and tissue in the human body to survive from the weakened treatment that is impacted by components of UPR.

\section{REFERENCES}

[1] Wu, J, and R J Kaufman. "From Acute Er Stress to Physiological Roles of the Unfolded Protein Response." Cell Death \& Differentiation, vol. 13, no. 3, 2006, pp. 374-384., doi:10.1038/sj.cdd.4401840.

[2] Chen, Xi, and Juan R. Cubillos-Ruiz. "Endoplasmic Reticulum Stress Signals in the Tumour and Its Microenvironment." Nature Reviews Cancer, vol. 21, no. 2, 2020, pp. 71-88., doi:10.1038/s41568020-00312-2.

[3] Salaroglio, Iris C., et al. "Perk Induces Resistance to Cell Death Elicited by Endoplasmic Reticulum Stress and Chemotherapy." Molecular Cancer, vol. 16, no. 1, 2017, doi:10.1186/s12943-017-0657-0.
[4] Wang, Miao, and Randal J. Kaufman. "The Impact of the Endoplasmic Reticulum Protein-Folding Environment on Cancer Development." Nature Reviews Cancer, vol. 14, no. 9, 2014, pp. 581-597., doi:10.1038/nrc3800.

[5] Cubillos-Ruiz, Juan R., et al. "Tumorigenic and Immunosuppressive Effects of Endoplasmic Reticulum Stress in Cancer." Cell, vol. 168, no. 4, 2017, pp. 692-706., doi:10.1016/j.cell.2016.12.004.

[6] Riha, Randal, et al. "Stressed out - Therapeutic Implications of Er Stress Related Cancer Research." Oncomedicine, vol. 2, 2017, pp. 156-167., doi: $10.7150 /$ oncm. 22477.

[7] Urra, Hery, et al. "Endoplasmic Reticulum Stress and the Hallmarks of Cancer." Trends in Cancer, vol. 2 , no. $5, \quad 2016, \quad$ pp. 252-262., doi:10.1016/j.trecan.2016.03.007. 